\author{
ANNALES \\ POLONICI MATHEMATICI \\ Online First version
}

\title{
On the behaviour of constants in some polynomial inequalities
}

\author{
Miroseaw Baran and Leokadia Bialas-Ciez (Kraków)
}

To the memory of Józef Siciak

\begin{abstract}
We study the asymptotical behaviour of optimal constants in the Hölder continuity property (HCP) of the Siciak extremal function and in the Vladimir Markov inequality equivalent to HCP. We observe that the optimal constants in polynomial inequalities of Markov and Bernstein type are related to some quantities that resemble capacities. We call them Hölder's and Markov's capacity and denote by $H(E), V(E)$ respectively. We compare these two capacities with the L-capacity $C(E)$. In particular, for any compact set $E \subset \mathbb{C}^{N}$ we prove the inequalities $V(E) \leq N C(E)$ and $H(E) \leq \sqrt{N} V(E)$. Moreover, we calculate the Markov capacity for polydiscs and rectangular prisms in $\mathbb{C}^{N}$ and we find that in these cases $V(E)=H(E)=C(E)$. Additionally, some new conditions equivalent to $\mathrm{HCP}$ and to the Andrey Markov inequality are given.
\end{abstract}

1. Introduction. Polynomial inequalities of Markov and Bernstein type are useful in the theory of approximation and numerical methods, especially in numerical solving of differential equations. Appropriate estimates of optimal constants in these inequalities lead to improved bounds of numerical errors. In general, finding the precise values of optimal constants for a given compact set $E \subset \mathbb{C}^{N}$ is a difficult task. In this paper we study the asymptotical behaviour of optimal constants in the Hölder continuity property (HCP) of the Siciak extremal function and in the Vladimir Markov inequality equivalent to $\mathrm{HCP}$.

Let $\mathcal{P}_{\nu}\left(\mathbb{C}^{N}\right)$ for $\nu=\left(\nu_{1}, \ldots, \nu_{N}\right) \in \mathbb{N}_{0}^{N}\left(\mathbb{N}_{0}:=\mathbb{N} \cup\{0\}, \mathbb{N}:=\{1,2, \ldots\}\right)$ be the vector space of polynomials $P=P\left(z_{1}, \ldots, z_{N}\right)$ with complex coefficients of degree at most $\nu_{i}$ with respect to $z_{i}$ for $i=1, \ldots, N$. The Siciak extremal

2010 Mathematics Subject Classification: 32U20, 32U35, 41A17.

Key words and phrases: Markov inequality, Bernstein inequality, Hölder property, Siciak extremal function, capacity.

Received 3 August 2018; revised 14 April 2019.

Published online *. 
function of a compact set $E \subset \mathbb{C}^{N}$ (see e.g. [28], [29]) is defined by

$$
\Phi(E, z):=\sup \left\{|P(z)|^{1 /|\nu|}: P \in \mathcal{P}_{\nu}\left(\mathbb{C}^{N}\right),|\nu| \geq 1,\|P\|_{E}=1\right\},
$$

where $\|\cdot\|_{E}$ is the supremum norm on $E$ and $|\nu|=\nu_{1}+\cdots+\nu_{N}$. The function $\Phi(E, \cdot)$ is known to satisfy the formula

$$
\Phi(E, z)=\lim _{n \rightarrow \infty} \phi_{n}(E, z)^{1 / n}=\sup _{n} \phi_{n}(E, z)^{1 / n}
$$

for $z \in \mathbb{C}^{N}$, where

$$
\phi_{n}(E, z):=\sup \left\{|P(z)|: P \in \mathcal{P}_{\nu}\left(\mathbb{C}^{N}\right), 1 \leq|\nu| \leq n,\|P\|_{E}=1\right\} .
$$

The function $\Phi(E, \cdot)$ is closely connected with the pluricomplex Green's function $V_{E}$ in view of the following formula proved by Zakharyuta and Siciak (see e.g. [21, Th. 5.1.7]):

$$
\Phi(E, z)=\exp V_{E}(z), \quad z \in \mathbb{C}^{N}
$$

where

$$
V_{E}(z):=\sup \left\{u(z): u \in \mathcal{L}_{N} \text { and } u \leq 0 \text { on } E\right\} \quad \text { for } z \in \mathbb{C}^{N},
$$

and $\mathcal{L}_{N}$ is the Lelong class of plurisubharmonic functions in $\mathbb{C}^{N}$ of logarithmic growth at infinity, i.e. $\mathcal{L}_{N}:=\left\{u \in \operatorname{PSH}\left(\mathbb{C}^{N}\right): u(z)-\log \|z\|_{2} \leq \mathcal{O}(1)\right.$ as $\left.\|z\|_{2} \rightarrow \infty\right\}$ and $\|z\|_{2}$ stands for the Euclidean norm in $\mathbb{C}^{N}$ (for background information, see [21]).

Let $V_{E}^{*}$ be the standard upper regularization of $V_{E}$. By Siciak's theorem, either $V_{E}^{*} \in \mathcal{L}_{N}$ or $V_{E}^{*} \equiv \infty$. This is equivalent to $E$ being non-pluripolar or pluripolar, respectively (non-polar or polar for $N=1$ ). For a non-polar set $E$, $V_{E}^{*}$ coincides with the Green's function $g_{E}$ of the unbounded component of $\hat{\mathbb{C}} \backslash E$ with logarithmic pole at infinity (as usual, $\hat{\mathbb{C}}=\mathbb{C} \cup\{\infty\}$ ). If we define the L-capacity of $E$ to be

$$
C(E):=\liminf _{\|z\|_{2} \rightarrow \infty} \frac{\|z\|_{2}}{\Phi^{*}(E, z)},
$$

then $E$ is a (pluri)polar set if and only if $C(E)=0$ (see [29]). Kołodziej [22] proved that $C(E)$ is a Choquet capacity in $\mathbb{C}^{N}$ (see also [23]). In the onedimensional space, $C(E)$ equals the logarithmic capacity of $E$. A compact set $E \subset \mathbb{C}^{N}$ is $L$-regular if $\lim _{w \rightarrow z} V_{E}^{*}(w)=0$ for every $z \in E$. Siciak proved that this property is equivalent to the continuity of $V_{E}$ in the whole space $\mathbb{C}^{N}$.

For multiindices $\alpha=\left(\alpha_{1}, \ldots, \alpha_{N}\right), \beta=\left(\beta_{1}, \ldots, \beta_{N}\right) \in \mathbb{N}_{0}^{N}$ we use the standard notations $D^{\alpha}=\frac{\partial^{|\alpha|}}{\partial z_{1}^{\alpha_{1}} \ldots \partial z_{N}^{\alpha_{N}}},|\alpha|=\alpha_{1}+\cdots+\alpha_{N}, \alpha !=\alpha_{1} ! \ldots \alpha_{N} !$, $\left(\begin{array}{l}\alpha \\ \beta\end{array}\right)=\left(\begin{array}{l}\alpha_{1} \\ \beta_{1}\end{array}\right) \ldots\left(\begin{array}{l}\alpha_{N} \\ \beta_{N}\end{array}\right), \beta^{\alpha}=\beta_{1}^{\alpha_{1}} \ldots \beta_{N}^{\alpha_{N}}$. The inequality $\alpha \leq \beta$ means that $\alpha_{1} \leq \beta_{1}, \ldots, \alpha_{N} \leq \beta_{N}$.

An important problem is the behaviour of the Siciak extremal function $\Phi(E, \cdot)$ in a neighbourhood of the set $E$. We are interested in seeing how some 
estimates of derivatives of polynomials on $E$ are related to the behaviour of $\Phi(E, \cdot)$. Note that the inequality

$$
\left\|D^{\nu} P\right\|_{E} \leq M^{|\nu|}|\nu|^{|\nu|}\|P\|_{E}
$$

with $M>0$ independent of $P \in \mathcal{P}_{\nu}\left(\mathbb{C}^{N}\right)$, is equivalent to the non-pluripolarity of $E$ (see [11]), i.e., to $\Phi(E, \cdot)$ being locally upper bounded. Moreover, the optimal constant $M$ in 1.2 is strictly related to the transfinite diameter and the Chebyshev constant of $E$. Another connection between the Siciak extremal function and the growth of derivatives of polynomials is given by the formula

$$
\Phi(E, z)=\limsup _{|\nu| \rightarrow \infty} M_{\nu}^{(\alpha)}(E, z)^{1 /|\nu|}
$$

where $M_{\nu}^{(\alpha)}(E, z):=\sup \left\{\left|D^{\alpha} P(z)\right|: P \in \mathcal{P}_{\nu}\left(\mathbb{C}^{N}\right),\|P\|_{E}=1\right\}$ for $z \in \mathbb{C}^{N}$ and $E$ is an L-regular set in $\mathbb{C}^{N}$ (see [10]). However, polynomial inequalities obtained from the above equality have constants that exponentially depend on the degree of polynomials. Therefore, these inequalities are less useful for applications.

Definition 1.1. We say that $E$ has the Hölder continuity property with exponent $\gamma \in(0,1]$ and constant $A>0$ (we write $E \in \operatorname{HCP}(\gamma, A)$ for short) if

$$
|\log \Phi(E, w)-\log \Phi(E, z)| \leq A|w-z|^{\gamma} \quad \text { for } w, z \in \mathbb{C}^{N}
$$

By an argument due to Błocki [30, Prop. 3.5], we need to verify 1.3 only for $w \in E$. In other words, $\operatorname{HCP}(\gamma, A)$ is equivalent to the inequality

$$
V_{E}(z) \leq A[\operatorname{dist}(z, E)]^{\gamma} \text { for } z \in \mathbb{C}^{N} \text {. }
$$

Taking into account (1.1), we can observe that the Hölder continuity property (1.3) is equivalent to the Bernstein-type inequality

$$
\|P\|_{E(r)} \leq \exp \left(A r^{\gamma}|\nu|\right)\|P\|_{E}
$$

for $P \in \mathcal{P}_{\nu}\left(\mathbb{C}^{N}\right)$, where $E(r):=\left\{z \in \mathbb{C}^{N}: \operatorname{dist}(z, E) \leq r\right\}$ for $r \in(0,1]$.

Definition 1.2. A compact set $E \subset \mathbb{C}^{N}$ satisfies the Vladimir Markov inequality with exponent $m \geq 1$ and constant $M>0(E \in \operatorname{VMI}(m, M)$ for short) if for every $\alpha, \nu \in \mathbb{N}_{0}^{N}$ and $P \in \mathcal{P}_{\nu}\left(\mathbb{C}^{N}\right)$,

$$
\left\|D^{\alpha} P\right\|_{E} \leq M^{|\alpha|} \frac{|\nu|^{m|\alpha|}}{(|\alpha| !)^{m-1}}\|P\|_{E} .
$$

The above inequality is strictly related to the estimate proved by Vladimir Markov for the interval $[-1,1] \subset \mathbb{C}$ :

$$
\left\|P^{(k)}\right\|_{[-1,1]} \leq \frac{n^{2}\left[n^{2}-1\right] \ldots\left[n^{2}-(k-1)^{2}\right]}{1 \cdot 3 \cdot \ldots \cdot(2 k-1)}\|P\|_{[-1,1]} \leq \frac{n^{2 k}}{k !}\|P\|_{[-1,1]}
$$


for $P \in \mathcal{P}_{n}(\mathbb{C})$ and $k \in \mathbb{N}$. Consequently, $[-1,1] \in \operatorname{VMI}(2,1)$. Property (1.5) for $|\alpha|=1$ is called the Andrey Markov inequality (AMI for short) and has recently been extensively studied (see e.g. [1]-[3], [14]-[17, [20], [27], 31-33]). In [5] we have proved that the Hölder continuity property is equivalent to the Vladimir Markov inequality on $E$. Moreover, the constants in $\mathrm{HCP}$ and VMI are closely related.

Theorem 1.3 ([5, Th. 2.9], [6, Cor. 18]). Let E be a compact subset of $\mathbb{C}^{N}$ and $\gamma \in(0,1], m \geq 1, A, M>0$. Then

$E \in \operatorname{HCP}(\gamma, A) \Longrightarrow E \in \operatorname{VMI}(m, M)$ with $m=1 / \gamma, M=\sqrt{N}(A \gamma e)^{1 / \gamma}$, $E \in \operatorname{VMI}(m, M) \Longrightarrow E \in \operatorname{HCP}(\gamma, A)$ with $\gamma=1 / m, A=M^{\gamma} N^{\gamma} m$.

Moreover, if $E \in \operatorname{VMI}(m, M)$ then $C(E) \geq \frac{1}{N M e^{m}}$, and if $E \in \operatorname{HCP}(\gamma, A)$ then $C(E) \geq \frac{1}{(A \gamma e)^{1 / \gamma}}$.

In this paper we study the optimal constants in the Hölder continuity property and in the Vladimir Markov inequality. The values of the exponents $\gamma$ and $m$ as well as of the constants $A$ and $M$ in $\operatorname{HCP}(\gamma, A)$ and $\operatorname{VMI}(m, M)$ are especially important in view of applications in numerical analysis and approximation (see e.g. [15], [19]). The best exponents in Markov-type inequalities have been calculated for some sets: see [26, Th. 15.3.5], [7], 8] and the references given therein. However, it is rarely possible to obtain exact values of optimal constants in these inequalities. Therefore, good estimates are of special importance. Our research is closely related to a result of Pommerenke [25] who discovered an elegant connection between the optimal constant in the Andrey Markov inequality with the logarithmic capacity for continua in the complex plane:

$$
\left\|P^{\prime}\right\|_{E} \leq \frac{2^{1 / n-1}}{C(E)} n^{2}\|P\|_{E} \quad \text { for } P \in \mathcal{P}_{n}(\mathbb{C})
$$

(this is a version proved by Eremenko [18, Th. 1]). Examining the asymptotical behaviour of the optimal constant in the Markov inequalities as the degree of the polynomials goes to infinity, we have observed that the limit is related to a quantity with properties typical of capacities. We call this quantity the Markov capacity of the given compact set.

Calculating the exact values of various kinds of capacities is a difficult task [13], [12], 22], [23], [4]. It is also hard to find the Markov and Hölder capacity for an arbitrary fixed compact set. We are able to calculate them mainly for polydiscs and rectangular prisms. However, we give some general estimates of these capacities.

In Section 2 we define the Markov capacity $V(E)$ of a compact set $E \subset \mathbb{C}^{N}$ and we recall the definition of the Hölder capacity $H(E)$ introduced in [6] in order to investigate optimal constants in HCP. 
The third section deals with basic properties of Markov and Hölder capacities. We prove a product formula for the Markov capacity. By means of this property, we calculate the Markov capacity for a polydisc $\left\{z \in \mathbb{C}^{N}\right.$ : $\left.\left|z_{1}-a_{1}\right| \leq r_{1}, \ldots,\left|z_{N}-a_{N}\right| \leq r_{N}\right\}$ and a rectangular prism $\left[a_{1}, b_{1}\right] \times \cdots \times$ $\left[a_{N}, b_{N}\right]$.

In Section 4 we show relations between Markov and Hölder capacities. We prove that $V(E) \leq N C(E)$ and $H(E) \leq \sqrt{N} V(E)$ (Proposition 4.1 and Theorem 4.2. Consequently, in the one-dimensional case we obtain $H(E) \leq$ $V(E) \leq C(E)$.

Section 5 is devoted to new conditions closely related to the Hölder continuity property and the Andrey Markov inequality. Although the conditions equivalent to $\mathrm{AMI}$ seem to be very similar to those equivalent to $\mathrm{HCP}$, the question of whether AMI implies HCP has remained unanswered for thirty years [24]. At the end of the paper, we present other open problems.

\section{Definition of Markov and Hölder capacities}

2.1. Markov capacity. We first give two conditions equivalent to the Vladimir Markov inequality.

Proposition 2.1. For any polynomial $P \in \mathcal{P}_{\nu}\left(\mathbb{C}^{N}\right)$ and $\alpha \in \mathbb{N}_{0}^{N}$, inequality (1.5) in the definition of $\operatorname{VMI}(m, M)$ implies

$$
\left\|D^{\alpha} P\right\|_{E} \leq\left(M_{1} \frac{|\nu|^{m}}{|\alpha|^{m-1}}\right)^{|\alpha|}\|P\|_{E}
$$

with $M_{1}=e^{m-1} M$. Next, inequality (2.1) implies

$$
\left\|D^{\alpha} P\right\|_{E} \leq M_{2}^{|\alpha|}|\alpha| !\left(\begin{array}{l}
|\nu| \\
|\alpha|
\end{array}\right)^{m}\|P\|_{E}
$$

with $M_{2}=e M_{1}$. In particular, (1.5) implies 2.2 with $M_{2}=e^{m}$ M. Moreover, 1.5 is a consequence of (2.2) with $M=M_{2}$.

Proof. Observe that for $a \geq b>c>0$ we have

$$
\frac{a}{b} \leq \frac{a-c}{b-c} \text {. }
$$

Consequently,

$$
\left(\begin{array}{l}
n \\
k
\end{array}\right)=\frac{n}{k} \cdot \frac{n-1}{k-1} \cdot \ldots \cdot \frac{n-k+1}{1} \geq\left(\frac{n}{k}\right)^{k} \quad \text { for all } k \leq n .
$$

From (2.3) and Stirling's estimate we have

$$
\begin{aligned}
M_{1} \frac{|\nu|^{m}}{|\alpha|^{m}}|\alpha| & \leq M_{1}\left(\begin{array}{c}
|\nu| \\
|\alpha|
\end{array}\right)^{m /|\alpha|}|\alpha|<M_{1}\left(\begin{array}{c}
|\nu| \\
|\alpha|
\end{array}\right)^{m /|\alpha|}\left(\frac{|\alpha| ! e^{|\alpha|}}{\sqrt{2 \pi|\alpha|}}\right)^{1 /|\alpha|} \\
& <e M_{1}\left(\begin{array}{c}
|\nu| \\
|\alpha|
\end{array}\right)^{m /|\alpha|}|\alpha| !^{1 /|\alpha|}
\end{aligned}
$$

and we see that 2.1) implies 2.2 with $M_{2}=e M_{1}$. 
Since

$$
\frac{M^{|\alpha|}}{|\alpha|^{\mid m-1}}<\frac{M^{|\alpha|} e^{|\alpha|(m-1)}}{\left(|\alpha|^{|\alpha|} \sqrt{2 \pi|\alpha|}\right)^{m-1}}<\left(e^{m-1} M\right)^{|\alpha|} \frac{1}{|\alpha|^{|\alpha|(m-1)}},
$$

we have $1.5 \Rightarrow 2.1$ with $M_{1}=e^{m-1} M$. Inequality 1.5 is an easy consequence of (2.2) with $M=M_{2}$.

We define the Markov capacity $V(E)$ using inequality (2.2). As usual, we adhere to the convention that $1 / \infty=0$.

Definition 2.2. Let $E$ be a compact subset of $\mathbb{C}^{N}$. The constant

$$
M_{\nu}(E, \alpha):=\sup \left\{\left\|D^{\alpha} P\right\|_{E}: P \in \mathcal{P}_{\nu}\left(\mathbb{C}^{N}\right),\|P\|_{E}=1\right\}, \quad \alpha, \nu \in \mathbb{N}_{0}^{N},
$$

is called the ( $\alpha, \nu)$-Markov constant of $E$ (see e.g. [32], [20], [10]). The Markov factor $M(m, E)$ of $E$ for exponent $m \geq 1$ is given by

$$
M(m, E):=\sup _{|\alpha| \geq 1} \sup _{\nu \geq \alpha}\left(\frac{M_{\nu}(E, \alpha)}{|\alpha| !\left(\begin{array}{l}
|\nu| \\
|\alpha|
\end{array}\right)^{m}}\right)^{1 /|\alpha|} .
$$

Let

$$
V_{m}(E):=\frac{1}{M(m, E)}=\inf _{|\alpha| \geq 1} \inf _{\nu \geq \alpha}\left\{\frac{[|\nu|(|\nu|-1) \cdots(|\nu|-|\alpha|+1)]^{m}}{|\alpha| !^{m-1} M_{\nu}(E, \alpha)}\right\}^{1 /|\alpha|} .
$$

We define the Markov capacity $V(E)$ by setting

$$
V(E):=\sup _{m \geq 1} V_{m}(E) .
$$

Observe that $V_{m}(E)>0$ (i.e. $\left.M(m, E)<\infty\right)$ is equivalent to the Vladimir Markov inequality with exponent $m$. Additionally, if $V_{m}(E)>0$ for some $m$ then $M(m, E)$ is the optimal constant in the Vladimir Markov inequality 2.2 with exponent $m$, i.e.

$$
\left.M(m, E)=\inf \left\{M_{2}>0: \text { inequality } 2.2\right) \text { is fulfilled }\right\} .
$$

By Proposition 2.1, also inequalities (1.5) and (2.1) hold with $M=M_{1}=$ $M(m, E)$, and so $E \in \operatorname{VMI}(m, M(m, E))$. Moreover, the condition $V(E)>0$ implies that

$$
m(E):=\inf \left\{m \in[1, \infty): V_{m}(E)>0\right\}
$$

is the best exponent in 1.5$), 2.1$ and 2.2 . Since the function

$$
(m(E), \infty) \ni m \mapsto M(m, E) \in \mathbb{R}
$$

is decreasing, we have

$$
V(E)=\frac{1}{\lim _{m \rightarrow \infty} M(m, E)}=\lim _{m \rightarrow \infty} V_{m}(E) .
$$


The best exponent in the Andrey Markov inequality is defined by

$$
\begin{aligned}
\mu(E) & :=\inf \left\{\mu \in[1, \infty): \sup _{|\alpha|=1 \nu \geq \alpha}\left(\frac{M_{\nu}(E, \alpha)}{|\alpha| !\left(\begin{array}{l}
|\nu| \\
|\alpha|
\end{array}\right)}\right)^{1 /|\alpha|}<\infty\right\} \\
& =\inf \left\{\mu \in[1, \infty): \sup _{|\alpha|=1} \sup _{\nu \geq \alpha} \frac{M_{\nu}(E, \alpha)}{|\nu|^{\mu}}<\infty\right\}
\end{aligned}
$$

and is usually called the Markov exponent for $E$ (see [8], 24]). We can easily see that

$$
\mu(E) \leq m(E) .
$$

Remark 2.3. If $E \subset \mathbb{R}^{N} \subset \mathbb{R}^{N}+i \mathbb{R}^{N}=\mathbb{C}^{N}$ then $\mu(E) \geq 2$ and so $m(E) \geq 2$. Therefore, for $E \subset \mathbb{R}^{N}$ it is sufficient to consider only $m \geq 2$.

2.2. Hölder capacity. To investigate the behaviour of $V_{E}$ and $\Phi(E, \cdot)$, we defined in [5] the radial modification of the pluricomplex Green's function and of the Siciak extremal function

$V_{E}^{\bullet}(r):=\sup \left\{V_{E}(x+w): x \in E,\|w\|_{2} \leq r\right\}, \quad \varphi_{E}^{\bullet}(r):=\exp V_{E}^{\bullet}(r), \quad r \geq 0$.

For a non-pluripolar compact set $E$, the function $V_{E}^{\bullet}$ has the following properties:

(a) $V_{a+\lambda E}^{\bullet}(r)=V_{E}^{\bullet}\left(\lambda^{-1} r\right)$ for $a \in \mathbb{C}^{N}$ and $\lambda>0$,

(b) $V_{E \times F}^{\bullet}(r)=\max \left\{V_{E}^{\bullet}(r), V_{F}^{\bullet}(r)\right\}$ for a compact set $F \subset \mathbb{C}^{N_{1}}$,

(c) $\lim _{r \rightarrow \infty}\left(V_{E}^{\bullet}(r)-\log r\right)=-\log C(E)$,

(d) $E \in \operatorname{HCP}(\gamma, A)$ if and only if

$$
V_{E}^{\bullet}(r) \leq A r^{\gamma} \quad \text { for } r \geq 0
$$

(see [5] for proofs).

Papers published over the last few years by Andrievskii, Carleson, Goncharov, Ransford, Rostand and Totik have shown an increasing interest in detailed investigation of the growth of Green's function in relation to the Markov constants (see e.g. [1]-[3], [9], [16, [17], [20], 27], 33], [31]). In order to study the best constant $A$ and the optimal exponent $\gamma$ in the Hölder continuity property of the pluricomplex Green's function $V_{E}$, we defined in [6] the Hölder capacity $H(E)$ as follows.

Definition 2.4. For $\gamma \in(0,1]$ we set

$$
H(E):=\sup _{0<\gamma \leq 1} H_{\gamma}(E) \quad \text { where } H_{\gamma}(E):=\left(\inf _{r>0} \frac{r^{\gamma}}{\gamma e V_{E}^{\bullet}(r)}\right)^{1 / \gamma} .
$$

Observe that $H_{\gamma}(E)>0$ is equivalent to the Hölder continuity property with exponent $\gamma$. Moreover, if $H(E)>0$ then

$$
\gamma(E):=\sup \left\{\gamma \in(0,1]: H_{\gamma}(E)>0\right\}
$$


is the best exponent in 2.5 and is called the Hölder exponent of $V_{E}$. By Theorem 1.3. $\gamma(E)=1 / m(E)$. Additionally, the constant

$$
A(\gamma, E):=\frac{1}{\gamma e H_{\gamma}(E)^{\gamma}}=\sup _{r>0} \frac{V_{E}^{\bullet}(r)}{r^{\gamma}}
$$

is the optimal constant in (1.3), (1.4) and 2.5).

3. Basic properties and examples. Some simple properties of Markov and Hölder capacities are listed below (cf. [6]).

REMARK 3.1. Let $E$ be a compact subset of $\mathbb{C}^{N}$. Then

- $V(a+\lambda E)=|\lambda| V(E)$ and $H(a+\lambda E)=|\lambda| H(E)$ for $a \in \mathbb{C}^{N}$ and $\lambda \in \mathbb{C} \backslash\{0\}$, - $V(F) \leq V(E)$ if $M_{\nu}(E, \alpha) \leq M_{\nu}(F, \alpha)$ for $\alpha, \nu \in \mathbb{N}_{0}^{N}$ and $E \subset \mathbb{C}^{N_{1}}$, $F \subset \mathbb{C}^{N_{2}}$ with two integers $N_{1}, N_{2}$ that can be distinct,

- $H(F) \leq H(E)$ if $V_{E}^{\bullet} \leq V_{F}^{\bullet}$ on $[0, \infty)$ and $E \subset \mathbb{C}^{N_{1}}, F \subset \mathbb{C}^{N_{2}}$ with two integers $N_{1}, N_{2}$ that can be distinct,

- $H_{\gamma}(E \times F)=\min \left\{H_{\gamma}(E), H_{\gamma}(F)\right\}$ and $H(E \times F)=\sup _{\gamma \in(0,1]} \min \left\{H_{\gamma}(E), H_{\gamma}(F)\right\}$.

Our principal tool in this section is a product property for Markov capacity. However, we are able to show it only in the case of $N$ compact subsets of $\mathbb{C}$. A general product property for Markov capacity would be of interest (cf. [13], [12], 4]).

Proposition 3.2. If $E=E_{1} \times \ldots \times E_{N} \subset \mathbb{C}^{N}$ then

$$
M(m, E)=\max _{j=1, \ldots, N} M\left(m, E_{j}\right) \text { and so } V(E)=\min _{j=1, \ldots, N} V\left(E_{j}\right) .
$$

Proof. The key observation here is a consequence of Vandermonde's convolution. Namely, the identity $\sum_{j=0}^{k}\left(\begin{array}{c}n \\ j\end{array}\right)\left(\begin{array}{c}m \\ k-j\end{array}\right)=\left(\begin{array}{c}n+m \\ k\end{array}\right)$ implies that

$$
\left(\begin{array}{c}
n \\
p
\end{array}\right)\left(\begin{array}{c}
m \\
q
\end{array}\right) \leq\left(\begin{array}{c}
n+m \\
p+q
\end{array}\right) \quad \text { for } p \in\{0, \ldots, n\}, q \in\{0, \ldots, m\}
$$

and thus

$$
\left(\begin{array}{l}
\nu \\
\alpha
\end{array}\right) \leq\left(\begin{array}{l}
|\nu| \\
|\alpha|
\end{array}\right) \quad \text { for } \alpha \leq \nu, \alpha, \nu \in \mathbb{N}_{0}^{N} .
$$

Next observe that $M_{\nu}(E, \alpha)=M_{\nu_{1}}\left(E_{1}, \alpha_{1}\right) \ldots M_{\nu_{N}}\left(E_{N}, \alpha_{N}\right)$ for $\alpha=$ $\left(\alpha_{1}, \ldots, \alpha_{N}\right), \nu=\left(\nu_{1}, \ldots, \nu_{N}\right)$ (see [10, Prop. 2.1]). From (3.1) we have

$$
\begin{aligned}
M(m, E) & \leq \sup _{|\alpha| \geq 1} \sup _{\nu \geq \alpha}\left(\frac{M_{\nu}(E, \alpha)}{\alpha !\left(\begin{array}{c}
\nu \\
\alpha
\end{array}\right)^{m}}\right)^{1 /|\alpha|} \\
& =\sup _{|\alpha| \geq 1} \sup _{\nu \geq \alpha}\left(\frac{M_{\nu_{1}}\left(E_{1}, \alpha_{1}\right)}{\alpha_{1} !\left(\begin{array}{c}
\nu_{1} \\
\alpha_{1}
\end{array}\right)^{m}} \ldots \frac{M_{\nu_{N}}\left(E_{N}, \alpha_{N}\right)}{\alpha_{N} !\left(\begin{array}{c}
\nu_{N} \\
\alpha_{N}
\end{array}\right)^{m}}\right)^{1 /|\alpha|} \\
& \leq \sup _{|\alpha| \geq 1} \sup _{\nu \geq \alpha}\left(M\left(m, E_{1}\right)^{\alpha_{1}} \ldots M\left(m, E_{N}\right)^{\alpha_{N}}\right)^{1 /|\alpha|} \leq \max _{j=1, \ldots, N} M\left(m, E_{j}\right) .
\end{aligned}
$$


It simplifies the notation, and causes no loss of generality, to assume that $\max _{j=1, \ldots, N} M\left(m, E_{j}\right)=M\left(m, E_{1}\right)$. It follows that

$$
\begin{aligned}
M(m, E) & \geq \sup _{\alpha=\left(\alpha_{1}, 0, \ldots, 0\right), \alpha_{1} \geq 1 \nu=\left(\nu_{1}, 0, \ldots, 0\right), \nu_{1} \geq \alpha_{1}}\left(\frac{M_{\nu}(E, \alpha)}{|\alpha| !\left(\begin{array}{l}
|\nu| \\
|\alpha|
\end{array}\right){ }^{m}}\right)^{1 /|\alpha|} \\
& =\sup _{\alpha_{1} \geq 1} \sup _{\nu_{1} \geq \alpha_{1}}\left(\frac{M_{\nu_{1}}\left(E, \alpha_{1}\right)}{\alpha_{1} !\left(\begin{array}{c}
\nu_{1} \\
\alpha_{1}
\end{array}\right)^{m}}\right)^{1 / \alpha_{1}}=M\left(m, E_{1}\right)=\max _{j=1, \ldots, N} M\left(m, E_{j}\right),
\end{aligned}
$$

and the proof is complete.

EXAMPLE 3.3. Using the classical Bernstein inequality for the unit disc $\mathbb{D}$ in the complex plane we get

$$
M_{n}(\mathbb{D}, k)=\frac{n !}{(n-k) !}, \quad n, k \in \mathbb{N}, k \leq n .
$$

As

$$
M(m, \mathbb{D})=\sup _{k \geq 1} \sup _{n \geq k}\left(\frac{n !}{(n-k) ! k !\left(\begin{array}{l}
n \\
k
\end{array}\right)^{m}}\right)^{1 / k}=\sup _{k \geq 1} \sup _{n \geq k}\left(\begin{array}{l}
n \\
k
\end{array}\right)^{-(m-1) / k}=1
$$

or $m \geq 1$ we see that the sequence $V_{m}(\mathbb{D})$ is constant and $V(\mathbb{D})=1$. Taking into account Remark 3.1, we obtain

$$
V(x+\rho \mathbb{D})=\rho=C(x+\rho \mathbb{D}) \text { for } x \in \mathbb{C}, \rho>0,
$$

and by Proposition 3.2 , for a polydisc $P(a, r)=\left\{z \in \mathbb{C}^{N}:\left|z_{1}-a_{1}\right| \leq\right.$ $\left.r_{1}, \ldots,\left|z_{N}-a_{N}\right| \leq r_{N}\right\}$ of polyradius $r=\left(r_{1}, \ldots, r_{N}\right) \in(0, \infty)^{N}$ and of center $a=\left(a_{1}, \ldots, a_{N}\right) \in \mathbb{C}^{N}$ we have

$$
V(P(a, r))=\min _{j=1, \ldots, N} r_{j}=V_{m}(P(a, r))=C(P(a, r)) .
$$

EXAMPLE 3.4. We will now calculate the Markov capacity for a rectangular prism $E=\left[a_{1}, b_{1}\right] \times \ldots \times\left[a_{N}, b_{N}\right] \subset \mathbb{R}^{N} \subset \mathbb{R}^{N}+i \mathbb{R}^{N}=\mathbb{C}^{N}$. Applying the Vladimir Markov inequality we get the formula

$$
M_{n}([-1,1], k)=T_{n}^{(k)}(1)=\frac{n^{2}\left[n^{2}-1\right] \ldots\left[n^{2}-(k-1)^{2}\right]}{1 \cdot 3 \cdot \ldots \cdot(2 k-1)}, \quad n, k \in \mathbb{N}, k \leq n,
$$

where $T_{n}(x)=\cos (n \arccos x)$ is the $n$th Chebyshev polynomial of the first kind (for $k=1$ it was proved by Andrey Markov). Observe that the sequence $a_{n}=\frac{(n+1) \ldots(n+k-1)}{(n-1) \ldots(n-k+1)}$ is decreasing for $n \geq k$. Indeed,

$$
\begin{aligned}
\frac{a_{n+1}}{a_{n}} & =\frac{(n+2) \ldots(n+k)}{n(n-1) \ldots(n-k+2)} \frac{(n-1) \ldots(n-k+1)}{(n+1) \ldots(n+k-1)} \\
& =\frac{(n+k)(n-k+1)}{(n+1) \cdot n} \leq 1
\end{aligned}
$$


for $n \geq k$. Consequently,

$$
\begin{aligned}
\frac{n^{2}\left[n^{2}-1\right] \ldots\left[n^{2}-(k-1)^{2}\right]}{[n(n-1) \ldots(n-(k-1))]^{2}} & =a_{n} \\
& \leq \frac{(k+1)(k+2) \ldots(2 k-1)}{(k-1) !}=\left(\begin{array}{c}
2 k-1 \\
k
\end{array}\right),
\end{aligned}
$$

and so

$$
n^{2}\left[n^{2}-1\right] \ldots\left[n^{2}-(k-1)^{2}\right] \leq\left(\begin{array}{c}
2 k-1 \\
k
\end{array}\right)\left(\begin{array}{l}
n \\
k
\end{array}\right)^{2} k !^{2} .
$$

Since $1 \cdot 3 \cdot \ldots \cdot(2 k-1)=\frac{(2 k-1) !}{2^{k-1}(k-1) !}$, we have

$$
T_{n}^{(k)}(1) \leq\left(\begin{array}{c}
2 k-1 \\
k
\end{array}\right)\left(\begin{array}{l}
n \\
k
\end{array}\right)^{2} k !^{2} \frac{2^{k-1}(k-1) !}{(2 k-1) !}=2^{k-1}\left(\begin{array}{l}
n \\
k
\end{array}\right)^{2} k ! .
$$

It follows that

$$
M(m,[-1,1]) \leq \sup _{k \geq 1}\left[\frac{2^{k-1}\left(\begin{array}{l}
n \\
k
\end{array}\right)^{2} k !}{k !\left(\begin{array}{l}
n \\
k
\end{array}\right)^{m}}\right]^{1 / k}=\sup _{k \geq 1} \sup _{n \geq k} \frac{2}{2^{1 / k}\left(\begin{array}{l}
n \\
k
\end{array}\right)^{(m-2) / k}}=2
$$

because we can take $m \geq 2$ (see Remark 2.3).

To estimate $M(m,[-1,1])$ from below, it is sufficient to take $n=k$. Indeed, as $T_{n}^{(n)}(1)=n ! 2^{n-1}$, we have

This finally yields

$$
M(m,[-1,1]) \geq \sup _{n \geq 1}\left(\frac{T_{n}^{(n)}(1)}{n !}\right)^{1 / n}=\sup _{n \geq 1} 2^{1-1 / n}=2 .
$$

$$
V([-1,1])=V_{m}([-1,1])=\frac{1}{M(m,[-1,1])}=\frac{1}{2} \quad \text { for any } m \geq 2,
$$

and by Remark 3.1 .

$$
V([a, b])=V_{m}([a, b])=\frac{b-a}{4}=C([a, b]) \quad \text { for } a, b \in \mathbb{R} .
$$

Therefore, by Proposition 3.2 .

$V\left(\left[a_{1}, b_{1}\right] \times \cdots \times\left[a_{N}, b_{N}\right]\right)=\frac{1}{4} \min _{j=1, \ldots, N}\left(b_{j}-a_{j}\right)=C\left(\left[a_{1}, b_{1}\right] \times \cdots \times\left[a_{N}, b_{N}\right]\right)$ (see [4]).

EXAMPLE 3.5. Some exact values of Hölder capacities are given in [6]:

- if $E$ is the unit ball in $\mathbb{C}^{N}$ (with respect to a given norm) then $H(E)=$ $C(E) H(\overline{\mathbb{D}})=C(E)$,

- if $E$ is a convex symmetric body in $\mathbb{R}^{N}$ then

$$
H(E)=2 C(E) H([-1,1])=C(E) .
$$

In particular, for a polydisc $E=\left\{z \in \mathbb{C}^{N}:\left|z_{1}-a_{1}\right| \leq r_{1}, \ldots,\left|z_{N}-a_{N}\right|\right.$ $\left.\leq r_{N}\right\}$ with $r=\left(r_{1}, \ldots, r_{N}\right) \in(0, \infty)^{N}$ and $\left(a_{1}, \ldots, a_{N}\right) \in \mathbb{C}^{N}$ we have

$$
H(E)=\min _{j=1, \ldots, N} r_{j}=C(E)=V(E) .
$$


Analogously, for a rectangular prism $E=\left[a_{1}, b_{1}\right] \times \cdots \times\left[a_{N}, b_{N}\right] \subset \mathbb{R}^{N} \subset$ $\mathbb{R}^{N}+i \mathbb{R}^{N}=\mathbb{C}^{N}$ we obtain

$$
H(E)=\frac{1}{4} \min _{j=1, \ldots, N}\left(b_{j}-a_{j}\right)=C(E)=V(E) .
$$

\section{Inequalities between Hölder, Markov and L-capacities}

4.1. Estimates of Markov and Hölder capacities. In [6] we have proved that for any compact set $E \subset \mathbb{C}^{N}$ the inequality $C(E) \geq H_{\gamma}(E)$ holds for all $\gamma \in(0,1]$. In particular,

$$
C(E) \geq H(E) .
$$

In this section we show estimates between $V(E)$ and $C(E)$ as well as $H(E)$ and $V(E)$.

Proposition 4.1. If $E$ is a compact subset of $\mathbb{C}^{N}$ and $m \geq 1$ then

$$
V_{m}(E) \leq N C(E) \text { and } V(E) \leq N C(E) .
$$

Proof. Fix $P \in \mathcal{P}_{\nu}(\mathbb{C})$ such that $\|P\|_{E}=1$. For $z \in E$ and $\zeta \in \mathbb{C}$ with $|\zeta| \leq r \in(0, \infty)$ we have

$$
\begin{aligned}
& |P(z+\zeta)|=\left|\sum_{\alpha \leq \nu} \frac{1}{\alpha !} D^{\alpha} P(z) \zeta^{\alpha}\right| \leq \sum_{\alpha \leq \nu} \frac{r^{|\alpha|}}{\alpha !}\left\|D^{\alpha} P\right\|_{E} \leq \sum_{\alpha \leq \nu} \frac{r^{|\alpha|}}{\alpha !} M_{\nu}(E, \alpha) \\
& \quad \leq \sum_{\alpha \leq \nu} \frac{r^{|\alpha|}}{\alpha !} M(m, E)^{|\alpha|}|\alpha| !\left(\begin{array}{c}
|\nu| \\
|\alpha|
\end{array}\right)^{m} \leq \sum_{l=0}^{|\nu|} r^{l} M(m, E)^{l} l !\left(\begin{array}{c}
|\nu| \\
l
\end{array}\right)^{m} \sum_{|\alpha|=l} \frac{1}{\alpha !} .
\end{aligned}
$$

Since $\sum_{|\alpha|=l} 1 / \alpha !=N^{l} / l !$, we get

$$
\begin{aligned}
|P(z+\zeta)| & \leq \sum_{l=0}^{|\nu|} r^{l} N^{l} M(m, E)^{l}\left(\begin{array}{c}
|\nu| \\
l
\end{array}\right)^{m} \\
& \leq\left(\sum_{l=0}^{|\nu|}\left(\begin{array}{c}
|\nu| \\
l
\end{array}\right)\left[r^{1 / m} N^{1 / m} M(m, E)^{1 / m}\right]^{l}\right)^{m} \\
& =\left(1+r^{1 / m} N^{1 / m} M(m, E)^{1 / m}\right)^{|\nu| m} .
\end{aligned}
$$

By the definition of the extremal function $\Phi(E, \cdot)$ and the Zakharyuta-Siciak formula (1.1), it follows that

$$
V_{E}^{\bullet}(r) \leq \log \left(1+r^{1 / m} N^{1 / m} M(m, E)^{1 / m}\right)^{m}
$$

and therefore

$$
\begin{aligned}
-\log C(E) & =\lim _{r \rightarrow \infty}\left(V_{E}^{\bullet}(r)-\log r\right) \\
& \leq \lim _{r \rightarrow \infty} \log \left(r^{-1 / m}+N^{1 / m} M(m, E)^{1 / m}\right)^{m}=\log (N M(m, E)) .
\end{aligned}
$$

Finally, we obtain 


$$
V_{m}(E)=1 / M(m, E) \leq N C(E),
$$

and this completes the proof.

TheOREM 4.2. Let $E$ be a compact subset of $\mathbb{C}^{N}$ and $m \geq 1$. Then

$$
H_{1 / m}(E) \leq \sqrt{N} V_{m}(E) \quad \text { and } \quad V_{m}(E) \leq N e^{m} H_{1 / m}(E) .
$$

Consequently,

$$
H(E) \leq \sqrt{N} V(E) .
$$

Proof. In order to prove the first inequality, fix $r>0$ and $P \in \mathcal{P}_{\nu}(\mathbb{C})$ such that $\|P\|_{E}=1$. Put $E_{r}:=\left\{z \in \mathbb{C}^{N}: \operatorname{dist}(z, E) \leq r\right\}$. Evidently, $\bigcup_{a \in E}\left\{z:\left|z_{j}-a_{j}\right| \leq r / \sqrt{N}, j=1, \ldots, N\right\} \subset E_{r}$, and so, by Cauchy's estimate and the Zakharyuta-Siciak formula (1.1), we have

$$
\begin{aligned}
\left\|D^{\alpha} P\right\|_{E} & \leq \frac{\alpha ! \sqrt{N}^{|\alpha|}}{r^{|\alpha|}}\|P\|_{E_{r}} \leq \frac{\alpha ! \sqrt{N}^{|\alpha|}}{r^{|\alpha|}} \exp \left(|\nu| V_{E}^{\bullet}(r)\right) \\
& \leq \frac{\alpha ! \sqrt{N}^{|\alpha|}}{r^{|\alpha|}} \exp \left(|\nu| A(1 / m, E) r^{1 / m}\right) .
\end{aligned}
$$

Therefore,

$$
\left\|D^{\alpha} P\right\|_{E} \leq \alpha ! \sqrt{N}^{|\alpha|} \inf _{r>0} \frac{\exp \left(|\nu| A(1 / m, E) r^{1 / m}\right)}{r^{|\alpha|}} .
$$

The infimum is achieved for $r=\left(\frac{|\alpha| m}{|\nu| A(1 / m, E)}\right)^{m}$ and we get

$$
\begin{aligned}
\left\|D^{\alpha} P\right\|_{E} & \leq \alpha ! \sqrt{N}|\alpha| \frac{(e|\nu| A(1 / m, E))^{|\alpha| m}}{(|\alpha| m)^{|\alpha| m}} \\
& =|\alpha| ! \sqrt{N}^{|\alpha|}\left(\frac{e A(1 / m, E)}{m}\right)^{|\alpha| m}\left(\frac{|\nu|}{|\alpha|}\right)^{|\alpha| m} .
\end{aligned}
$$

From (2.3) we have

$$
M_{\nu}(E, \alpha) \leq|\alpha| ! \sqrt{N}^{|\alpha|}\left(\frac{e A(1 / m, E)}{m}\right)^{|\alpha| m}\left(\begin{array}{l}
|\nu| \\
|\alpha|
\end{array}\right)^{m} .
$$

Consequently,

$$
M(m, E) \leq \sqrt{N}\left(\frac{e A(1 / m, E)}{m}\right)^{m}
$$

and so

$$
V_{m}(E)=1 / M(m, E) \geq \sqrt{N}^{-1} H_{1 / m}(E),
$$

which completes the proof of the first estimate in the theorem.

To show the second bound, we use inequality (4.1) to get

$$
V_{E}^{\bullet}(r) \leq \log \left(1+r^{1 / m} N^{1 / m} M(m, E)^{1 / m}\right)^{m} \leq m r^{1 / m} N^{1 / m} M(m, E)^{1 / m} .
$$

This yields $A(1 / m, E) \leq m N^{1 / m} M(m, E)^{1 / m}$, and so $N e^{m} H_{1 / m}(E) \geq$ $V_{m}(E)$. 
Corollary 4.3. In the one-dimensional case we have

$$
C(E) \geq V(E) \geq H(E) \text {. }
$$

\section{New conditions equivalent to HCP and to the Andrey Markov} inequality. For a non-pluripolar compact set $E \subset \mathbb{C}^{N}$ the radial modification of the Siciak extremal function $\varphi_{E}^{\bullet}$ defined in $(2.4)$ is increasing in $[0, \infty)$ and the mapping $\mathbb{R} \ni t \mapsto \log \varphi_{E}^{\bullet}\left(e^{t}\right)$ is convex. Moreover,

$$
\lim _{r \rightarrow \infty} \frac{r}{\varphi_{E}^{\bullet}(r)}=C(E)
$$

and $\varphi_{E}^{\bullet}$ is continuous on $[0, \infty)$ if and only if $E$ is L-regular. Set $\mathcal{P}_{n}\left(\mathbb{C}^{N}\right):=$ $\bigcup_{|\nu| \leq n} \mathcal{P}_{\nu}\left(\mathbb{C}^{N}\right)$.

Our starting point in this section is the equivalence given below that is easy to prove by means of Taylor's formula and Cauchy's inequality. Fix $m \geq 1$ and $P \in \mathcal{P}_{n}\left(\mathbb{C}^{N}\right)$. The Pleśniak-type inequality

$$
|P(z)| \leq e^{k}\|P\|_{E} \quad \text { for } k \in\{0, \ldots, n\}, \operatorname{dist}(z, E) \leq(k / n)^{m}
$$

implies the Vladimir Markov inequality

$$
\left\|D^{\alpha} P\right\|_{E} \leq \frac{e^{|\alpha|} n^{m|\alpha|}}{|\alpha| !^{m-1}}\|P\|_{E} \quad \text { for } \alpha \in \mathbb{N}_{0}^{N} .
$$

Conversely, for $M>0$ the inequality

$$
\left\|D^{\alpha} P\right\|_{E} \leq \frac{M^{|\alpha|} n^{m|\alpha|}}{|\alpha| !^{m-1}}\|P\|_{E} \quad \text { for } \alpha \in \mathbb{N}_{0}^{N}
$$

implies the property

$$
|P(z)| \leq e^{k}\|P\|_{E} \quad \text { for } k \in\{0, \ldots, n\}, \operatorname{dist}(z, E) \leq \frac{1}{M N}\left(\frac{k}{n}\right)^{m} .
$$

We will consider a property slightly more general than (5.1) and (5.3). Let $f:(0,1] \rightarrow(0, \infty)$ be a continuous function with $\sup \left\{f(x)^{x}: x \in(0,1]\right\}<\infty$.

Proposition 5.1. If $E \subset \mathbb{C}^{N}$ is a compact set such that

$$
\left\|D^{\alpha} P\right\|_{E} \leq|\alpha| ! f\left(\frac{|\alpha|}{n}\right)^{|\alpha|}\|P\|_{E}, \quad P \in \mathcal{P}_{n}\left(\mathbb{C}^{N}\right), \alpha \in \mathbb{N}_{0}^{N}
$$

then

$$
\varphi_{E}^{\bullet}(r) \leq \sup \left\{f(x)^{x}(N r)^{x}: x \in(0,1]\right\} .
$$

In the case of Vladimir Markov inequality (1.5) (equivalent to (2.1)), we can take $f(x)=M / x^{m}$ and inequality (5.5) implies the estimate

$$
\log \varphi_{E}^{\bullet}(r) \leq \frac{m(M N)^{1 / m}}{e} r^{1 / m} \quad \text { for } r \in(0,1]
$$

that gives a bound different from the one proved in [5, Th. 2.9]. 
Proof. Fix $P \in \mathcal{P}_{n}\left(\mathbb{C}^{N}\right), r \in(0,1]$ and $z \in \mathbb{C}^{N}$ such that $\operatorname{dist}(z, E) \leq r$. We have

$$
\begin{aligned}
\frac{|P(z)|}{\|P\|_{E}} & \leq \sum_{|\alpha| \leq n} \frac{1}{\alpha !} \frac{\left\|D^{\alpha} P\right\|_{E}}{\|P\|_{E}} r^{|\alpha|} \leq \sum_{|\alpha| \leq n} \frac{1}{\alpha !}|\alpha| ! f\left(\frac{|\alpha|}{n}\right)^{|\alpha|} r^{|\alpha|} \\
& =\sum_{k=0}^{n} N^{k} f\left(\frac{k}{n}\right)^{k} r^{k}=\sum_{k=0}^{n}\left[N^{k / n} f\left(\frac{k}{n}\right)^{k / n} r^{k / n}\right]^{n} \\
& \leq(n+1) \sup \left\{f(x)^{x}(N r)^{x}: x \in(0,1]\right\}^{n}
\end{aligned}
$$

and inequality $(5.5)$ is proved.

Now consider a (strictly) increasing continuous function $\psi:[0,1] \rightarrow$ $[0, \infty)$ such that $\psi(0)=0$.

Proposition 5.2. Let $E$ be an L-regular compact set in $\mathbb{C}^{N}$. Then the Pleśniak-type property

$$
|P(z)| \leq e^{k}\|P\|_{E} \quad \text { for } P \in \mathcal{P}_{n}\left(\mathbb{C}^{N}\right), k \in\{0, \ldots, n\}, \operatorname{dist}(z, E) \leq \psi(k / n)
$$

is equivalent to

$$
\log \varphi_{E}^{\bullet}(r) \leq \psi^{-1}(r) \quad \text { for all } r \in[0, \psi(1)] .
$$

Proof. Inequality (5.7) implies that $\left(|P(z)| /\|P\|_{E}\right)^{1 / n} \leq \exp \left(\psi^{-1}(\psi(k / n))\right)$ $=\exp (k / n)$. In order to prove the converse, fix $r \in(0, \psi(1)]$ (for $r=0$ inequality (5.7) is obvious) and an arbitrary $\ell \geq 1$. Set $s:=\psi^{-1}(r) \in(0,1]$ and take an integer $n>\ell / s$. We can find $k \in\{1, \ldots, n\}$ such that

$$
\frac{k-1}{n}<s \leq \frac{k}{n} \text {. }
$$

From this it follows that $k \geq \ell+1$ and $r \leq \psi(k / n)$. Fix $P \in \mathcal{P}_{n}\left(\mathbb{C}^{N}\right)$ and $z \in \mathbb{C}^{N} \backslash E$ such that $r=\operatorname{dist}(z, E)$. By the assumption and (5.8),

$$
\begin{aligned}
\left(\frac{|P(z)|}{\|P\|_{E}}\right)^{1 / n} & \leq \exp \left(\frac{k}{n}\right)=\exp \left(\frac{k}{k-1} \frac{k-1}{n}\right) \\
& \leq \exp \left(\frac{\ell+1}{\ell} s\right)=\exp \left(\frac{\ell+1}{\ell} \psi^{-1}(r)\right) .
\end{aligned}
$$

Taking the sup over all $P \in \mathcal{P}_{n}\left(\mathbb{C}^{N}\right)$ of the left hand side and letting $n \rightarrow \infty$, we get

$$
\Phi(E, z) \leq \exp \left(\frac{\ell+1}{\ell} \psi^{-1}(r)\right) .
$$

Now we can let $\ell \rightarrow \infty$ and obtain

$$
\Phi(E, z) \leq \exp \left(\psi^{-1}(\operatorname{dist}(z, E))\right) .
$$

Consequently, if we take the sup over all $z \in \mathbb{C}^{N} \operatorname{such}$ that $\operatorname{dist}(z, E) \leq r$, we get inequality (5.7). 
Corollary 5.3 ([5, Th. 2.9]). If the set E satisfies Vladimir Markov inequality (5.2) then

$$
\log \varphi_{E}^{\bullet}(r) \leq(M N)^{1 / m} r^{1 / m} \quad \text { for } r \in(0,1] .
$$

Corollary 5.4. Any L-regular compact set $E \subset \mathbb{C}^{N}$ has the Pleśniaktype property 5.6 with $\psi=\left(\log \varphi_{E}^{\bullet}\right)^{-1}$. Moreover, if $f:[0, \infty) \rightarrow[0, \infty)$ is an increasing continuous function such that $f(0)=0$ and $\log \varphi_{E}^{\bullet}(r) \leq f(r)$ for $r \in\left[0, f^{-1}(1)\right]$ then $E$ has property (5.6) with $\psi=f^{-1}$.

REMARK 5.5. Inequality (5.7) is equivalent to

$$
\log \varphi_{E}^{\bullet}(\psi(s)) \leq s \quad \text { for all } s \in[0,1] .
$$

Consider the function $\phi_{n}(E, \cdot)$ defined in the first section and

$$
\varphi_{n}^{\bullet}(r):=\sup \left\{\phi_{n}(E, w+r \zeta): w \in E,\|\zeta\|_{2} \leq 1\right\}, \quad r \in[0, \infty) .
$$

Proposition 5.6. For a fixed $m \geq 1$ the Hölder continuity property with exponent $1 / \mathrm{m}$ is equivalent to the following condition:

$$
\sup \left\{\frac{1}{k} \log \varphi_{n}^{\bullet}\left(\left(\frac{k}{n}\right)^{m}\right): n \in \mathbb{N}, k \in\{1, \ldots, n\}\right\}<\infty,
$$

and consequently to

$$
\sup \left\{\frac{\log \varphi_{n}^{\bullet}\left(x^{m}\right)}{n x}: x \in[1 / n, 1], n \in \mathbb{N}\right\}<\infty .
$$

On the other hand, the Andrey Markov inequality with exponent $m$ is equivalent to

$$
\sup \left\{\log \varphi_{n}^{\bullet}\left((1 / n)^{m}\right): n \in \mathbb{N}\right\}<\infty .
$$

Proof. The equivalence $(5.9) \Leftrightarrow(5.10)$ is easy to show. The property (5.11) is equivalent to the Andrey Markov inequality by the classical Pleśniak estimate (see [24]). We will merely prove the equivalence between $\operatorname{HCP}(1 / m)$ and (5.9). First assume (5.9). Set

$$
C:=\sup \left\{\frac{1}{k} \log \varphi_{n}^{\bullet}\left(\left(\frac{k}{n}\right)^{m}\right): n \in \mathbb{N}, k \in\{1, \ldots, n\}\right\} .
$$

In order to prove $\mathrm{HCP}(1 / m)$ it is sufficient to show the Pleśniak-type inequality (5.1) with a constant $M$ instead of $e$, because it is equivalent to the Vladimir Markov inequality and thus to the Hölder continuity property. Fix $n, P \in \mathcal{P}_{n}\left(\mathbb{C}^{N}\right), k \in\{1, \ldots, n\}$ and $z \in \mathbb{C}^{N} \backslash E$ such that $\operatorname{dist}(z, E) \leq(k / n)^{m}$. We have

$$
|P(z)| \leq \phi_{n}(E, z)\|P\|_{E} \leq \varphi_{n}^{\bullet}\left((k / n)^{m}\right)\|P\|_{E} \leq M^{k}\|P\|_{E}
$$

with $M=e^{C}$. The converse is easy to prove by means of property 5.1 with some positive constant $M$ instead of $e$. 
Corollary 5.7. The Hölder continuity property (1.4) with exponent $\gamma=$ $1 / m$ is equivalent to the condition

$$
\sup \left\{\frac{1}{t} \log \varphi_{E}^{\bullet}\left(t^{m}\right): t \in(0,1]\right\}<\infty .
$$

Since $r \mapsto \varphi_{E}^{\bullet}(r)$ is continuous for $r>0$ (see [5]), the above property is equivalent to

$$
\limsup _{t \rightarrow 0^{+}} \frac{1}{t} \log \varphi_{E}^{\bullet}\left(t^{m}\right)<\infty .
$$

The above two conditions seem to be very close to 5.11. However, the question of whether the Andrey Markov inequality implies the Hölder continuity property remains open (see e.g. [24]).

\subsection{Open problems}

- The Andrey Markov inequality need not be fulfilled with the Markov exponent $\mu(E)$, as was shown in [7]. Is it true that $M(m(E), E)<\infty$ ? The same question for $A(\gamma(E), E)$.

- In Proposition 3.2 we have the product formula for the Markov capacity of $N$ subsets of the complex plane. Is it also true for two arbitrary compact sets $E \subset \mathbb{C}^{N_{1}}, F \subset \mathbb{C}^{N_{2}}$ ? The same question for the Hölder capacity is also interesting.

- In Section 3 we have observed that for polydiscs and rectangular prisms the Markov capacity is equal to the Hölder one. Is this true in general? Other sets with exact values of Markov and Hölder capacities would also be interesting.

Acknowledgements. The work was partially supported by the National Science Centre (NCN), Poland No. 2013/11/B/ST1/03693 and 2017/ 25/B/ST1/00906.

\section{References}

[1] M. Altun and A. Goncharov, On smoothness of the Green function for the complement of a rarefied Cantor-type set, Constr. Approx. 33 (2011), 265-271.

[2] V. V. Andrievskii, On the Green function for a complement of a finite number of real intervals, Constr. Approx. 20 (2004), 565-583.

[3] V. V. Andrievskii, On optimal smoothness of the Green function for the complement of a Cantor-type set, Constr. Approx. 24 (2006), 113-122.

[4] M. Baran and L. Bialas-Ciez, Product property for capacities in $\mathbb{C}^{N}$, Ann. Polon. Math. 106 (2012), 19-29.

[5] M. Baran and L. Bialas-Ciez, Hölder continuity of the Green function and Markov brothers' inequality, Constr. Approx. 40 (2014), 121-140.

[6] M. Baran and L. Bialas-Ciez, Hölder continuity of the Green function, Markov-type inequality and a capacity related to HCP, Dolomites Res. Notes Approx. 7 (2014), $16-21$. 
[7] M. Baran, L. Bialas-Ciez and B. Milówka, On the best exponent in Markov inequality, Potential Anal. 38 (2013), 635-651.

[8] M. Baran and W. Pleśniak, Markov's exponent of compact sets in $\mathbb{C}^{n}$, Proc. Amer. Math. Soc. 123 (1995), 2785-2791.

[9] L. Bialas-Ciez, Smoothness of Green's functions and Markov-type inequalities, in: Banach Center Publ. 92, Inst. Math., Polish Acad. Sci., 2011, 27-36.

[10] L. Bialas-Ciez, Siciak's extremal function via Bernstein and Markov constants for compact sets in $\mathbb{C}^{N}$, Ann. Polon. Math. 106 (2012), 41-51.

[11] L. Bialas-Ciez and M. Jędrzejowski, Transfinite diameter of Bernstein sets in $\mathbb{C}^{N}$, J. Inequal. Appl. 7 (2002), 393-404.

[12] Z. Błocki, A. Edigarian and J. Siciak, On the product property for the transfinite diameter, Ann. Polon. Math. 101 (2011), 209-214.

[13] T. Bloom et J.-P. Calvi, Sur le diamètre transfini en plusieurs variables, C. R. Acad. Sci. Paris 329 (1999), 567-570.

[14] L. P. Bos and P. D. Milman, Sobolev-Gagliardo-Nirenberg and Markov type inequalities on subanalytic domains, Geom. Anal. Funct. Anal. 5 (1995), 853-923.

[15] J.-P. Calvi and N. Levenberg, Uniform approximation by discrete least squares polynomials, J. Approx. Theory 152 (2008), 82-100.

[16] L. Carleson and V. Totik, Hölder continuity of Green's functions, Acta Sci. Math. (Szeged) 70 (2004), 557-608.

[17] S. Celik and A. Goncharov, Smoothness of the Green function for a special domain, Ann. Polon. Math. 106 (2012), 113-126.

[18] A. Eremenko, A Markov-type inequality for arbitrary plane continua, Proc. Amer. Math. Soc. 135 (2007), 1505-1510.

[19] M. Gentile, A. Sommariva and M. Vianello, Polynomial interpolation and cubature over polygons, J. Comput. Appl. Math. 235 (2011), 5232-5239.

[20] A. Goncharov, Weakly equilibrium Cantor-type sets, Potential Anal. 40 (2014), 143161.

[21] M. Klimek, Pluripotential Theory, London Math. Soc. Monogr. N.S. 6, Clarendon Press, Oxford, 1991.

[22] S. Kołodziej, The logarithmic capacity in $\mathbb{C}^{n}$, Ann. Polon. Math. 48 (1988), 253-267.

[23] S. Kołodziej, Capacities associated to the Siciak extremal function, Ann. Polon. Math. 49 (1989), 279-290.

[24] W. Pleśniak, Markov's inequality and the existence of an extension operator for $C^{\infty}$ functions, J. Approx. Theory 61 (1990), 106-117.

[25] Ch. Pommerenke, On the derivative of a polynomial, Michigan Math. J. 6 (1959), 373-375.

[26] Q. I. Rahman and G. Schmeisser, Analytic Theory of Polynomials, Oxford Sci. Publ., Oxford, 2002.

[27] T. Ransford and J. Rostand, Hölder exponents of Green's functions of Cantor sets, Comput. Methods Funct. Theory 8 (2008), 151-158.

[28] J. Siciak, On some extremal functions and their applications in the theory of analytic functions of several complex variables, Trans. Amer. Math. Soc. 105 (1962), 322-357.

[29] J. Siciak, Extremal plurisubharmonic functions in $\mathbb{C}^{N}$, Ann. Polon. Math. 39 (1981), $175-211$.

[30] J. Siciak, Wiener's type sufficient conditions in $\mathbb{C}^{N}$, Univ. Iagel. Acta Math. 35 (1997), 151-161.

[31] F. Toókos and V. Totik, Markov inequality and Green functions, Rend. Circ. Mat. Palermo (2) Suppl. 76 (2005), 91-102.

[32] V. Totik, On Markoff's inequality, Constr. Approx. 18 (2002), 427-441. 
[33] V. Totik, Metric properties of harmonic measures, Mem. Amer. Math. Soc. 184 (2006), no. 867, vi+163 pp.

Mirosław Baran

Leokadia Bialas-Ciez

Faculty of Mathematics, Physics and Technical Science Institute of Mathematics

Pedagogical University of Cracow

Jagiellonian University

Łojasiewicza 6

Podchorążych 2

30-084 Kraków, Poland

30-348 Kraków, Poland

E-mail: miroslaw.baran@up.krakow.pl

E-mail: leokadia.bialas-ciez@uj.edu.pl 